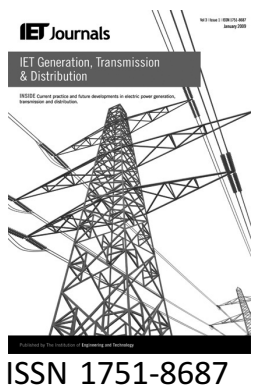

\title{
Choice of estimator for distribution system state estimation
}

\author{
$\begin{array}{lll}\text { R. Singh } & \text { B.C. Pal } & \text { R.A. Jabr } \\ & \end{array}$ \\ ${ }^{1}$ Department of Electrical and Electronic Engineering, Imperial College, London, UK \\ ${ }^{2}$ Electrical Computer and Communication Engineering Department, Notre Dame University, Zouk Mosbeh, Lebanon \\ E-mail: b.pal@imperial.ac.uk
}

Abstract: In this study, a statistical framework is introduced to assess the suitability of various state estimation (SE) methodologies for the purpose of distribution system state estimation (DSSE). The existing algorithms adopted in the transmission system SE are reconfigured for the distribution system. The performance of three SE algorithms has been examined and discussed in standard 12-bus and 95-bus UK-GDS network models.

\section{Nomenclature}

$m, n \quad$ number of measurements and state variables

$\boldsymbol{x}_{t}, \hat{\boldsymbol{x}} \quad$ true state and estimated state vectors, respectively $(n \times 1)$

$\boldsymbol{P}_{x}, \hat{\boldsymbol{P}}_{x} \quad$ numerically computed and estimated error covariance matrices, respectively $(n \times n)$

$E[\cdot] \quad$ expectation operator

$\epsilon \quad$ normalised state error squared variable

$\boldsymbol{z} \quad$ measurement vector $(m \times 1)$

$\boldsymbol{b}(\boldsymbol{x}) \quad$ expectation of measurement vector $(m \times 1)$

$\sigma_{z i} \quad$ standard deviation of the $i$ th measurement

$\boldsymbol{R}_{\boldsymbol{z}} \quad$ measurement error covariance matrix $(m \times m)$

$\boldsymbol{e}_{\boldsymbol{z}} \quad$ measurement error vector $(m \times 1)$

$r_{i} \quad$ normalised residual of $i$ th measurement

\section{Introduction}

Deregulation of power system and the introduction of distributed generation (DG) to distribution networks has challenged the operational philosophy of the distribution systems. The passive nature of the network can only accommodate restricted amount of DG capacity. This means that significant network reinforcement will be necessary to accommodate DG and load growth in the future. An alternative would be to change the approach to network operation such as introducing control to distribution network operation. A range of technology innovations is needed to change the way distribution systems operate. The innovations must pin down on new architecture for distribution network control centre with performance critical software functions like state estimation (SE), optimal power flow (OPF) and network-specific sensor placement and integration.

In transmission systems, $\mathrm{SE}$ is a fairly routine task and a host of established methodologies exist [1]. These cannot simply be transferred to distribution systems because the planning, design and operation philosophy of distribution networks are different from those in the transmission networks. The distribution network topology and characteristics are different and most importantly the amount of available network measurements is very limited. The $\mathrm{SE}$ methodologies adopted in transmission systems start showing their limitations when exposed to the specifics of distribution networks [2].

Furthermore, the potential benefits of using SE technologies in distribution network control have not been explored mainly because of the absence of adequate network measurements and also the lack of rigorous methodology and tools that could be applied on restricted measurements. The development of new distribution system state estimation (DSSE) is a challenging task as the tools to evaluate the quality of SE must consider a number of issues relating to measurement types, locations and numbers.

Methodologies on which such tools could be built are not available at present. However, some interesting research has 
been done in DSSE [3-10]. Lu et al. [3] propose a threephase DSSE algorithm. The algorithm uses a currentbased formulation of the weighted least-squares (WLS) method in which the power measurements, current measurements and voltage measurements are converted to their equivalent currents, and the Jacobian terms are constant and equal to the admittance matrix elements. The observability analysis of the proposed distribution system is also discussed. Lin and Teng [4] have proposed a new fast decoupled state estimator with equality constraints. The proposed method is based on the equivalent current measurement in rectangular coordinates. Baran and Kelley [5] have introduced a computationally efficient algorithm based on branch currents as state variables. The method is demonstrated to work well in radial and weakly meshed systems. This concept is further refined by Wang and Schulz [6] and they have presented a revised branch current-based DSSE algorithm. In this algorithm, the load estimated at every node from an automated metre reading system is used as a pseudo measurement. $\mathrm{Li}$ [7] has presented a distribution system state estimator based on WLS approach and three-phase modelling techniques. Li has also demonstrated the impact of the measurement placement and measurement accuracy on the estimated results. A rule-based approach for measurement placement is presented by Baran et al. [8]. Ghosh et al. [9] have presented an alternative approach to DSSE using a probabilistic extension of the radial load flow algorithm treating the real measurements as solution constraints. The algorithm that accounts for non-normally distributed loads, incorporates the concept of load diversity and can interact with a load allocation routine. The field results are discussed in [10].

The DSSE literature is either based on the probabilistic load flow or direct adaptation of transmission system SE algorithms (particularly WLS). The issue of measurement inadequacy is addressed through pseudo measurements that are stochastic in nature. However, the performance of the SE algorithms under the stochastic behaviour of pseudo measurements is not addressed in the DSSE literature.

The work presented in this paper investigates the existing transmission system SE techniques and algorithms and assesses their suitability to the DSSE problem. The selected algorithms are tested on the 12-bus and 95-bus UK-GDS network models against some statistical measures like bias, consistency and overall quality of the estimates. Unlike many other distribution systems, the UK distribution network is fairly balanced and that has prompted us to go with a singlephase approach, although the method is generic. Furthermore, the statistical measures utilised in this paper mainly depend on the probability distribution of the measurements and not on the line model of the network. Following this introduction, a theoretical framework for the statistical measures is established in Section 2. The consistency and the quality of the estimates utilise the asymptotic state error covariance matrix. The various $\mathrm{SE}$ techniques along with the details of their state error covariance matrices are discussed in Section 3. The efficacy of the algorithms is examined on standard test systems and discussed in Section 4.

\section{Statistical measures}

In distribution systems, measurements are predominantly of pseudo type, which are statistical in nature, so the performance of a state estimator should be based on some statistical measures. Various statistical measures such as bias, consistency and quality have been adopted for assessing the effectiveness of SE in other technology areas such as target tracking [11]. We explore these for the DSSE applications. Briefly we describe the statistical measures as follows.

\subsection{Bias}

A state estimator is said to be unbiased if the expected value of error in the state estimate is zero. Mathematically an unbiased estimator can be defined as

$$
E\left[\left(\boldsymbol{x}_{t}-\hat{\boldsymbol{x}}\right)\right]=\mathbf{0}
$$

\subsection{Consistency}

If the error in an estimate statistically corresponds to the corresponding covariance matrix then the estimate (and hence the technique generating this estimate) is said to be consistent. One measure of consistency is the normalised state error squared variable

$$
\boldsymbol{\epsilon}=\left(\boldsymbol{x}_{t}-\hat{\boldsymbol{x}}\right)^{\mathrm{T}} \hat{\boldsymbol{P}}_{\boldsymbol{x}}^{-1}\left(\boldsymbol{x}_{t}-\hat{\boldsymbol{x}}\right)
$$

where, $\hat{\boldsymbol{P}}_{x}$, denotes the estimated state error covariance matrix.

For the estimator to be consistent $\epsilon$ should be within its confidence bounds, which can be obtained from the error statistics.

2.2.1 Choice of confidence regions: In the univariate case when the estimation error is represented by a normal distribution with zero mean and known variance, one can use the tables of normal distribution to compute the confidence intervals. However, in the multivariate case when the estimation error is represented by a normal distribution with zero mean vector and known covariance matrix, such confidence intervals are difficult to compute because tables are available only for the bivariate case. Alternatively, one could setup limits for each component on the basis of distribution, but this procedure has the disadvantages that the choice of limit is somewhat arbitrary and in some cases leads to tests that may be poor against some alternatives. Moreover, such limits are difficult to compute. The procedure given below, which is based on $\chi^{2}$-statistics, can be easily computed and applied in the multivariate case. Furthermore, it can be theoretically justified based on the following lemma. The proof the lemma can be found in [12]. 
Lemma 1: If an $n$-component vector $v$ is distributed according to $\mathcal{N}(\boldsymbol{0}, \boldsymbol{T})$ (non-singular), then $\boldsymbol{v}^{\mathrm{T}} \boldsymbol{T}^{-1} \boldsymbol{v}$ is distributed according to $\chi^{2}$-distribution with $n$ degrees of freedom.

\subsection{2 $\chi^{2}$-statistics: It can be shown that if the errors} in measurements are normally distributed, the SE error corresponding to these measurements will be normally distributed with zero mean vector and covariance matrix given by $E\left[\left(\boldsymbol{x}_{t}-\hat{\boldsymbol{x}}\right)\left(\boldsymbol{x}_{t}-\hat{\boldsymbol{x}}\right)^{\mathrm{T}}\right]$. By utilising this fact and Lemma 1, the normalised squared error $\epsilon$ (2) should follow a $\chi^{2}$-distribution with $n$ degrees of freedom for a consistent estimator, where $n$ is the number of states. In other words for the estimator to be consistent, $\epsilon$ should lie within its confidence bounds that can be obtained from the standard $\chi^{2}$-table for a chosen confidence level $\alpha$. Lower and upper bounds for this confidence level can be given by $\chi_{n}^{2}((1-\alpha) / 2)$ and $\chi_{n}^{2}((1+\alpha) / 2)$, respectively. In statistics, a $95 \%$ confidence interval is considered to be adequate.

\subsection{3 $\chi^{2}$-test over Monte Carlo simulations: In} practice, statistical tests are performed using a number of Monte Carlo simulations. Consider the system has $n$ number of states and $M$ is the number of Monte Carlo simulations, then the normalised squared error follows a $\chi^{2}$-distribution with $M n$ degrees of freedom. Mathematically

$$
E[\epsilon]=\frac{\chi_{M n}^{2}(\alpha)}{M}
$$

For large number of Monte Carlo runs $\chi_{M n}^{2}(\alpha) \simeq M n$, which results in

$$
E[\epsilon]=n
$$

Hence the mean of $\epsilon$ should approach to the number of states with the increase in the number of simulations.

\subsection{Quality}

Quality of an estimate is inversely related to its variance. For the multivariate case, the square root of the determinant of the error covariance matrix measures the volume of $1-\sigma$ ellipsoid and is used here to quantify the total variance of an estimate. Hence, the quality of the estimate can be defined as

$$
Q_{\operatorname{det}}=\log \left(\frac{1}{\sqrt{\operatorname{det}\left(\boldsymbol{P}_{x}\right)}}\right)
$$

Sometimes, in large networks, it becomes difficult to compute the determinant of the error covariance matrix numerically because of precision limits of the solver. In this situation, an alternate way to define the quality is to use the trace of the error covariance matrix. However, this ignores the off-diagonal information. The quality as function of the trace of the error covariance matrix can be written as

$$
Q_{\text {trace }}=\log \left(\frac{1}{\operatorname{tr}\left(\boldsymbol{P}_{x}\right)}\right)
$$

\section{State estimation techniques}

Various algorithms have been suggested for transmission system SE [1]. All these algorithms work well in transmission systems because there is high redundancy in the measurements. However, in distribution systems, because of sparsity of measurements, there is less or no redundancy in the measurements. Hence, when these algorithms are exposed to distribution systems they start showing their limitations. For example, in transmission systems, weighted least absolute value estimator (WLAV) eliminates bad data out of redundant measurements, but in distribution systems it fails to work because it treats every pseudo measurement as bad data and there is no redundancy to eliminate these pseudo measurements.

This section briefly explains the most common SE techniques to examine their suitability for the DSSE problem under stochastic behaviour of the pseudo measurements and limited or no redundancy. All these techniques use the following measurement model.

\subsection{Measurement model}

$$
\boldsymbol{z}=\boldsymbol{h}(\boldsymbol{x})+\boldsymbol{e}_{z}
$$

where, $\boldsymbol{e}_{z} \sim \mathcal{N}\left(\boldsymbol{0}, \boldsymbol{R}_{z}\right)$ is zero mean Gaussian noise with error covariance matrix $\boldsymbol{R}_{z}\left(=\operatorname{diag}\left\{\sigma_{z 1}^{2}, \sigma_{z 2}^{2}, \ldots, \sigma_{z m}^{2}\right\}\right)$. We define the normalised residual of $i$ th measurement $r_{i}$ as

$$
r_{i}=\frac{z_{i}-h_{i}(\boldsymbol{x})}{\sigma_{z i}}
$$

where, $r_{i} \sim \mathcal{N}(0,1)$. The class of estimators discussed in this section are based on maximum likelihood theory. They rely on a priori knowledge of the distribution of the measurement error (Gaussian in this case, with zero mean and known covariance). A generalised estimation problem seeks to minimise the following objective

$$
J=\sum_{i=1}^{m} \rho\left(r_{i}\right)
$$

The different estimators can be characterised based on the choice of the $\rho$ function.

\subsection{Weighted least squares estimation}

WLS is a quadratic form of the maximum likelihood estimation problem. The WLS problem can be stated as the minimisation of the following objective function

$$
\frac{1}{2}[z-h(x)]^{\mathrm{T}} \boldsymbol{R}_{z}^{-1}[\boldsymbol{z}-\boldsymbol{h}(\boldsymbol{x})]
$$


The above objective takes the form given in (9) for

$$
\rho\left(r_{i}\right)=\frac{1}{2} r_{i}^{2}
$$

An estimate of state was obtained iteratively using the Newton method according to

$$
\hat{\boldsymbol{x}}_{k+1}=\hat{\boldsymbol{x}}_{k}+\left(\boldsymbol{H}^{\mathrm{T}}\left(\hat{\boldsymbol{x}}_{k}\right) \boldsymbol{R}_{\boldsymbol{z}}^{-1} \boldsymbol{H}\left(\hat{\boldsymbol{x}}_{k}\right)\right)^{-1} \boldsymbol{H}^{\mathrm{T}}\left(\hat{\boldsymbol{x}}_{k}\right) \boldsymbol{R}_{\boldsymbol{z}}^{-1}\left[\boldsymbol{z}-\boldsymbol{b}\left(\hat{\boldsymbol{x}}_{k}\right)\right]
$$

where

$$
\boldsymbol{H}\left(\hat{\boldsymbol{x}}_{k}\right)=\left[\frac{\partial \boldsymbol{b}(\boldsymbol{x})}{\partial \boldsymbol{x}}\right]_{x=\hat{\boldsymbol{x}}_{k}}
$$

\subsection{Weighted least absolute value estimator}

WLAV estimator is based on the minimisation of the $L_{1}$ norm of weighted measurement residual, and can be expressed as

$$
\left\|\boldsymbol{R}_{\boldsymbol{z}}^{-1 / 2}[\boldsymbol{z}-\boldsymbol{b}(\boldsymbol{x})]\right\|_{1}
$$

which is equivalent to (9) when

$$
\rho\left(r_{i}\right)=\left|r_{i}\right|
$$

Existing techniques use linear programming or interior point methods to solve this problem. In this paper we have used a primal dual interior point method [13].

\subsection{Schweppe Huber generalised $M$ (SHGM) estimator}

This estimator combines both WLS and WLAV estimators. The $\rho$ function for SHGM estimator is given by

$$
\rho\left(r_{i}\right)=\left\{\begin{array}{ccc}
\frac{1}{2} r_{i}^{2} & \text { if } & \left|r_{i}\right| \leq a \omega_{i} \\
a \omega_{i}\left|r_{i}\right|-\frac{1}{2} a^{2} \omega_{i}^{2} & \text { otherwise }
\end{array}\right.
$$

The performance of this estimator highly depends upon the weight factor $\omega_{i}$ and tuning parameter $a$. In this paper, the solution to this problem was obtained using iteratively reweighted least squares (IRLS) method [1]. The parameter $a=1.5$ was used in simulations.

3.4.1 State error covariance matrix: An estimate of the asymptotic covariance matrix at convergence can be expressed as $[14,15]$

$$
\hat{\boldsymbol{P}}_{\boldsymbol{x}}=\alpha\left(\boldsymbol{H}^{\mathrm{T}}(\hat{\boldsymbol{x}}) \boldsymbol{R}_{\boldsymbol{z}}^{-1} \boldsymbol{H}(\hat{\boldsymbol{x}})\right)^{-1}
$$

where $\hat{\boldsymbol{x}}=\lim _{k} \hat{\boldsymbol{x}}_{k}$. The value of $\alpha$ depends on the choice of the estimator. An expression for $\alpha$ is given by [14]

$$
\alpha=\frac{E\left[\psi^{2}(r)\right]}{\left(E\left[\psi^{\prime}(r)\right]\right)^{2}}
$$

where $\psi(r)=\partial \rho(r) / \partial r$ and $\psi^{\prime}(r)=\partial \psi(r) / \partial r$.

The numerical computation of $\alpha$ for various estimators is given in the Appendix. Table 1 summarises the various estimators used in this paper. Table 1 indicates a typical value of $\alpha$ for SHGM considering $a \omega_{i}=1.5$. Since the IRLS method is used for SHGM, the value of $\alpha$ changes during the estimation process depending upon the weight $\omega_{i}$.

\section{Case study}

The algorithms discussed in the previous section were applied on a 12-bus radial distribution network model and on a part of the UK generic distribution system model (95-bus UKGDS). Figs. 1 and 2 show the schematic of the test systems. Network and load data for these networks can be found in [16] and [17], respectively.

\subsection{State variables}

The bus voltage magnitudes and angles were considered as state variables except at the reference bus (Bus \#1) for which the bus angle was assumed to be zero. Hence, the number of states to be evaluated was 23 and 189 for the 12-bus test system and the UK-GDS, respectively.

\subsection{Measurements}

It was assumed that the errors associated with the measurements are independent identically distributed (i.i.d.). Three types of measurements were taken into consideration. The telemetered measurements were utilised as real measurements. Zero injections with a very low

Table 1 State estimators: summary

\begin{tabular}{|l|c|c|}
\hline & Solution for $\hat{\boldsymbol{x}}$ & Asymptotic error covariance $\hat{\boldsymbol{P}}_{\boldsymbol{x}}$ \\
\hline WLS & Newton & $\left(\boldsymbol{H}^{\top}(\hat{\boldsymbol{x}}) \boldsymbol{R}_{\mathbf{z}}^{-1} \boldsymbol{H}(\hat{\boldsymbol{x}})\right)^{-1}$ \\
\hline WLAV & PDIP & $\frac{\pi}{2}\left(\boldsymbol{H}^{\top}(\hat{\boldsymbol{x}}) \boldsymbol{R}_{\boldsymbol{z}}^{-1} \boldsymbol{H}(\hat{\boldsymbol{x}})\right)^{-1}$ \\
\hline SHGM & IRLS & $1.037\left(\boldsymbol{H}^{\top}(\hat{\boldsymbol{x}}) \boldsymbol{R}_{\mathbf{z}}^{-1} \boldsymbol{H}(\hat{\boldsymbol{x}})\right)^{-1 a}$ \\
\hline
\end{tabular}

${ }^{\mathrm{a}} a=1.5, \omega_{i}=1$

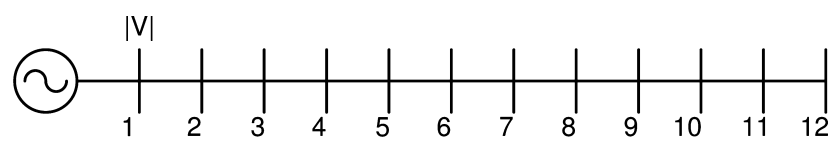

Figure 1 Twelve-bus test system 


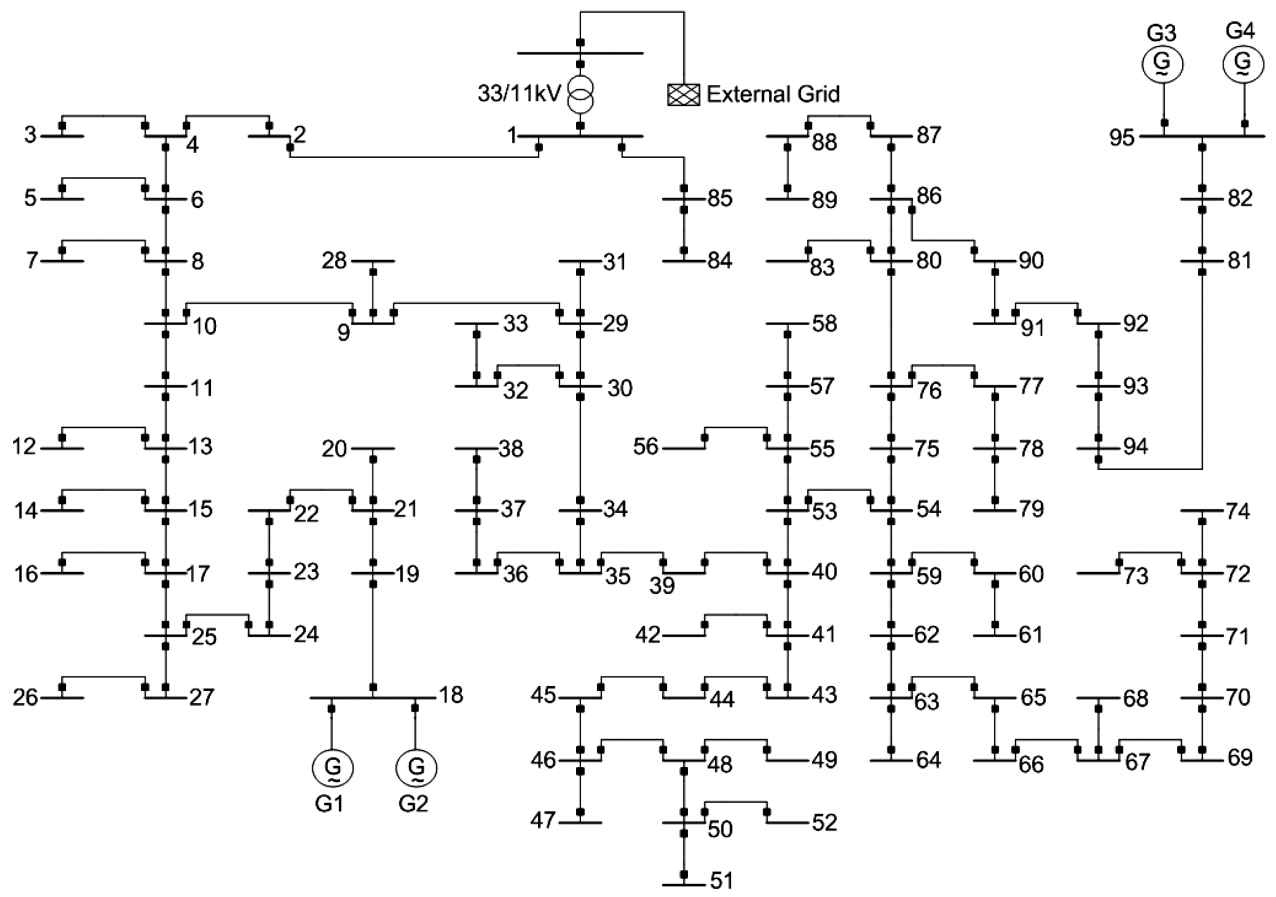

Figure 2 UK-GDS: 95-bus test system

variance $\left(10^{-8}\right)$ were modelled as virtual measurements. Loads were modelled as pseudo measurements. Various scenarios considering the errors in real measurements as 1 and $3 \%$, whereas 20 and $50 \%$ in pseudo measurements were examined. The range of error in pseudo measurements was chosen on the basis of errors in load estimates of various classes of customers, like industrial, domestic and commercial. The loads of the industrial customers can be estimated more accurately than the domestic and commercial, thus they have less error. On the other hand, loads of domestic customers are difficult to estimate, hence they have large error. The error in commercial load estimates lies between the two. It was also taken into consideration that with this choice of range, the maximum demand limits at various buses are not violated and the condition of linear approximation is valid. The mean value for these measurements was obtained using distribution system load flow. Table 2 summarises the measurements and their redundancy level for the two test network models.

\subsection{Measurement variance}

A $\pm 3 \sigma$ deviation around the mean covers more than $99.7 \%$ area of the Gaussian curve. Hence, for a given \% of maximum error about mean $\mu_{z i}$, the standard deviation of error was computed as follows

$$
\sigma_{z i}=\frac{\mu_{z i} \times \% \text { error }}{3 \times 100}
$$

The square of standard deviation gives the variance of the measurement.

\subsection{Simulation results}

The performance of the estimators was evaluated for the following cases:

Case 1: Error in real measurement $1 \%$ and pseudo measurement $20 \%$

Table 2 Measurements used in study

\begin{tabular}{|c|c|c|c|}
\hline Test system & Real measurements $\left(m_{r}\right)$ & $\begin{array}{l}\text { Virtual \& pseudo } \\
\text { measurements }\left(m_{p}\right)\end{array}$ & $\begin{array}{l}\text { Redundency } \\
\left(m_{r}+m_{p}\right) / n\end{array}$ \\
\hline twelve-bus & $3\left(V_{1}, P_{1-2}, Q_{1-2}\right)$ & $\begin{array}{c}22 \text { (loads only no zero } \\
\text { injections) }\end{array}$ & $\frac{25}{23}=1.09$ \\
\hline $\begin{array}{l}\text { UK-GDS (a) limited } \\
\text { redundancy }\end{array}$ & $5\left(V_{1}, P_{1-2}, Q_{1-2}, P_{1-85}, Q_{1-85}\right)$ & $\begin{array}{c}188 \text { (loads and zero } \\
\text { injections) }\end{array}$ & $\frac{193}{189}=1.02$ \\
\hline $\begin{array}{l}\text { UK-GDS (b) } \\
\text { increased } \\
\text { redundancy }\end{array}$ & $\begin{array}{l}21\left(V_{1}, V_{18}, V_{19}, V_{20}, V_{21}, V_{95}, P_{1-2}, Q_{1-2}\right. \\
P_{1-85}, Q_{1-85}, P_{18-19}, Q_{18-19}, P_{82-95}, Q_{82-95} \\
\left.P_{15-17}, Q_{15-17}, P_{34-35}, Q_{34-35}, \delta_{19}, \delta_{20}, \delta_{21}\right)\end{array}$ & $\begin{array}{l}188 \text { (loads and zero } \\
\text { injections) }\end{array}$ & $\frac{209}{189}=1.11$ \\
\hline
\end{tabular}


Case 2: Error in real measurement $1 \%$ and pseudo measurement $50 \%$

Case 3: Error in real measurement 3\% and pseudo measurement $20 \%$

Case 4: Error in real measurement 3\% and pseudo measurement $50 \%$

4.4.1 Twelve-bus system: In the 12-bus test system, the voltage magnitude measurement at bus \#1 and power flow measurement in line \#1-2 were considered as real measurement. Fig. 3 shows the variation of the expected value of the normalised state error squared with various Monte Carlo steps for the 12-bus distribution system. It is clear from the figure that as the number of Monte Carlo steps increases, the expected value of normalised state error square variable approaches the number of states, which agrees with (4). Also after 400 Monte Carlo steps, the error in $E[\epsilon]$ is within $1 \%$ of the number of states. Hence, we chose 400 Monte Carlo steps for the simulations. A larger number of Monte Carlo steps gives slightly better results but it increases the computation time.

Fig. 4 shows the error plots with the number of simulations for the three estimators. The plots shown are for the worst case scenario (Case 4), that is, the error associated with real measurements is $3 \%$ and that with pseudo measurements is $50 \%$. The estimation errors for all the states are displayed in Fig. 4, however, they are indistinguishable because of the overlaps. It is evident from the figure that the error varies about zero mean. This indicates that all the three estimators are unbiased. It was also found that for all other cases, the three estimators were unbiased.

Figs. 5-8 show the consistency plots for the estimators for cases 1-4. A 95\% confidence level was used to define the confidence bounds. It was found that WLS shows consistent results in all test cases. On the other hand, WLAV is
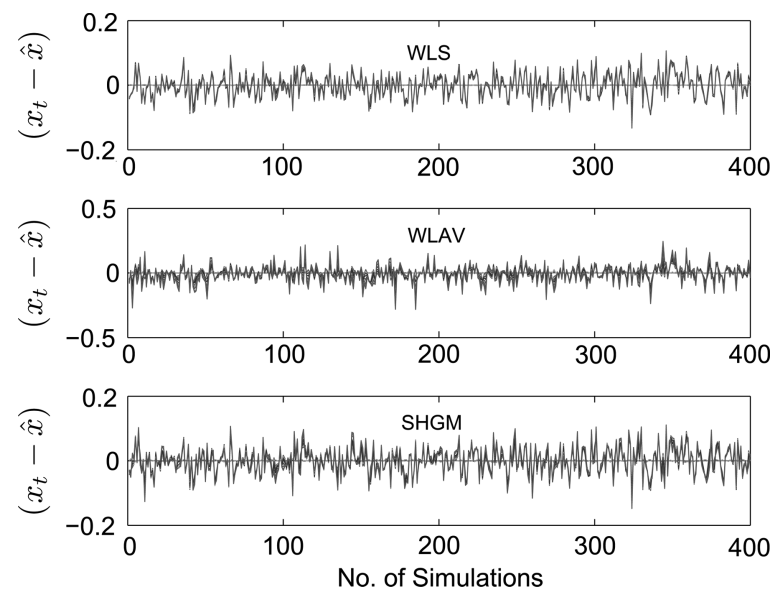

Figure 4 Twelve-bus system estimation error plot for all state variables: error in true measurements $=3 \%$, error in pseudo measurements $=50 \%$

inconsistent in all the cases. It is interesting to note that SHGM is inconsistent for small errors in pseudo measurements and consistent for large errors in pseudo measurements. The reason is that the measurement set considered for study is predominantly comprised of the pseudo measurements, and large error in pseudo measurements increases the measurement variance (19). Also the computation of variance in (19) is based on the maximum error. This results in low normalised residual $\left(\left|r_{i}\right|\right)$ for pseudo measurements. Owing to this fact the normalised residual becomes less than the cutoff value $a \omega_{i}(16)$, and the estimator behaves like WLS. However, this is not always true. Whenever the normalised residual exceeds the cutoff value, the estimator becomes inconsistent. It will be shown that for the 95-bus UK-GDS system, SHGM becomes inconsistent for these cases of large errors too.

Table 3 shows the performance summary of the 12-bus test system. Two types of qualities are shown. As expected, the

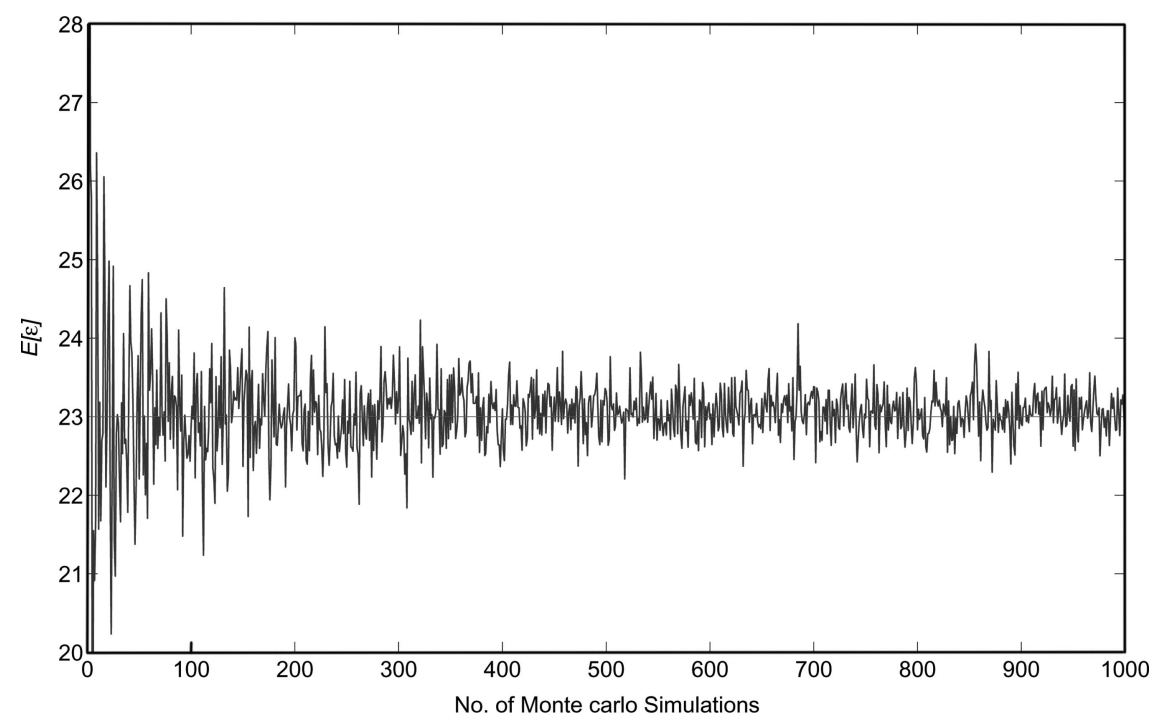

Figure 3 Variation of $E[\epsilon]$ with different Monte Carlo steps 

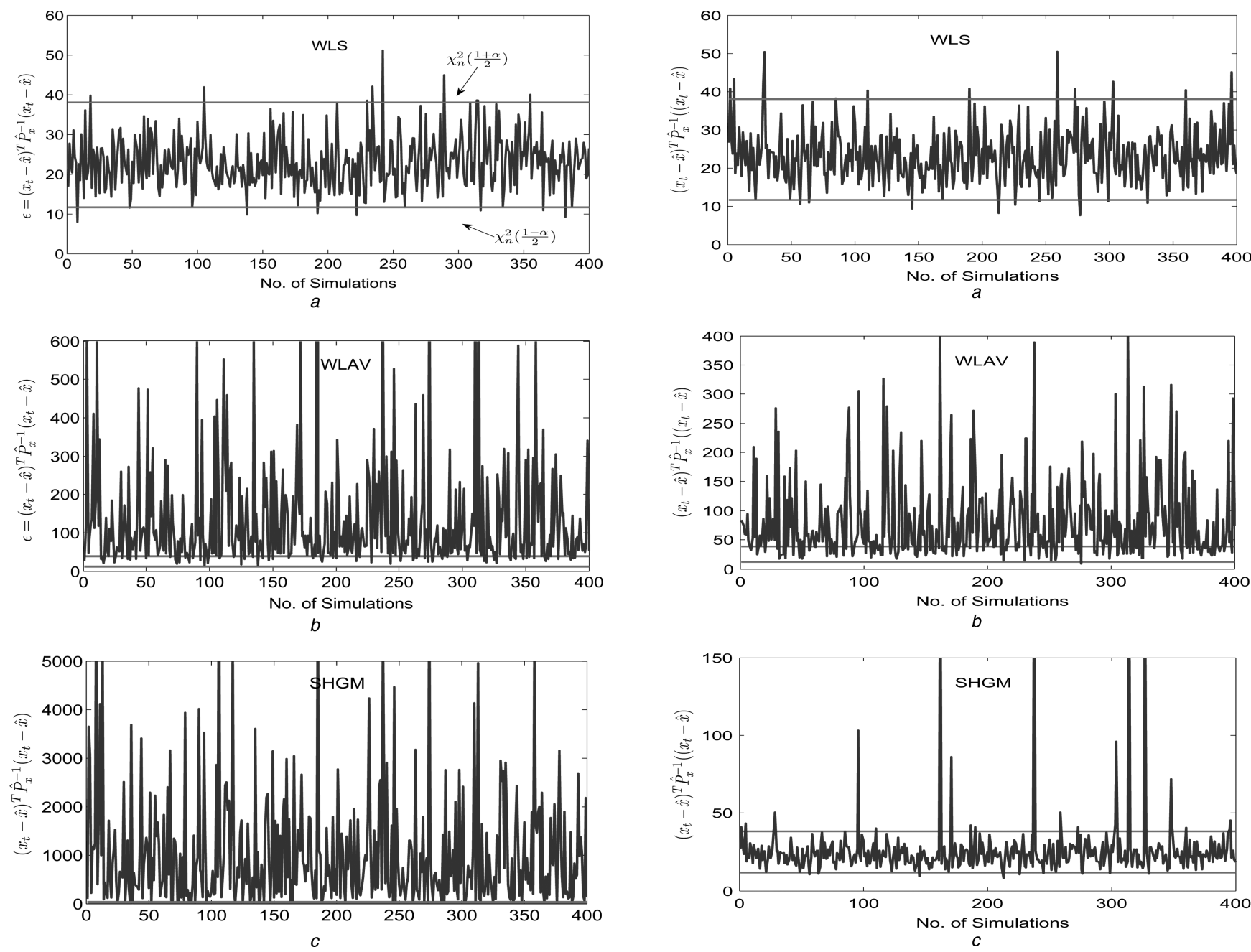

Figure 5 Twelve-bus system consistency plot: error in true measurements $=1 \%$, error in pseudo measurements $=20 \%$

quality of the estimates decreases with the increase in the error in measurements. This decrease is significant with the increase in error in the real measurements as compared to the pseudo measurements.

4.4.2 Ninety-five-bus UK-GDS: The performance of the estimators was also evaluated on the 95-bus test system model for all the test cases analysed in the 12-bus test system. It was observed that in the 95-bus test system also, 400 Monte Carlo steps are sufficient to bring down the error in $E[\epsilon]$ within $1 \%$ of the number of states. The following two cases were considered:

\section{(a) Limited redundancy}

In this case, the real measurements were considered to be available at the main substation. Hence, the voltage magnitude measurement at bus \#1 and power flow measurements in lines \#1-2 and \#1-85 were taken as real measurements. It was observed that in the 95-bus test system all the estimators were unbiased. However, only

Figure 6 Twelve-bus system consistency plot: error in true measurements $=1 \%$, error in pseudo measurements $=50 \%$

WLS was found to be consistent in all the test cases. Hence, the consistency plots of WLS in all four test cases are displayed in Fig. 9. The consistency plot for SHGMis also shown in Fig. 10 for the test Case 2. It is clear from Fig. 10 that the SHGM which was consistent in Case 2 in the 12-bus system no longer remains consistent in larger systems.

(a) Increased redundancy

In this case, the redundancy was increased by placing the measurements at DG locations first and then measurements were placed at optimal locations. The optimality criterion and details of the measurement placement appear in [18]. Furthermore, the phasor measurements were also deployed at optimally selected buses. The real measurement set in this study consists of the following measurements:

1. Voltage measurements at buses \#1, \#18, \#19, \#20, \#21 and \#95 

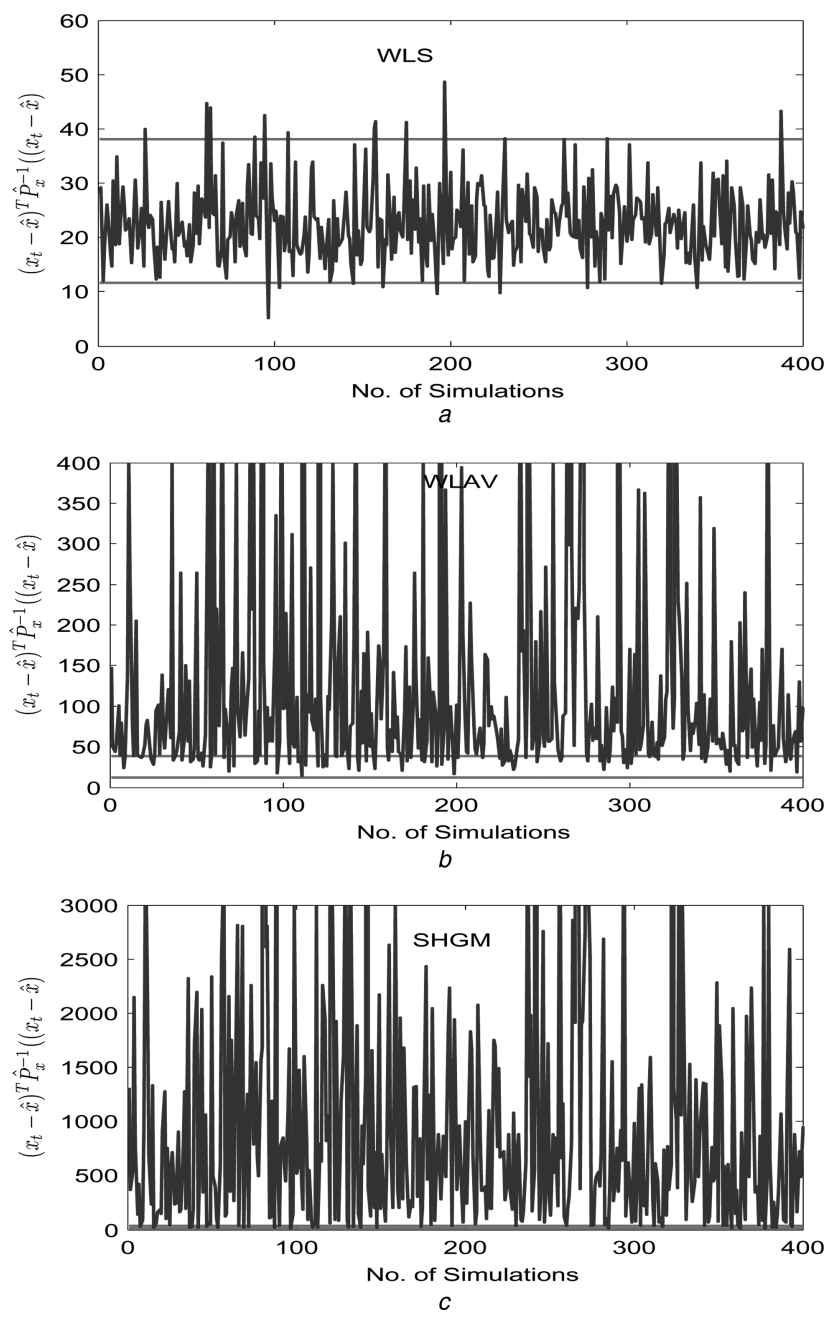

Figure 7 Twelve-bus system consistency plot: error in true measurements $=3 \%$, error in pseudo measurements $=20 \%$

2. Line flow measurements in lines \#1-2, \#1-85, \#82-95, \#18-19, \#15-17 and \#34-35

3. Phasor measurements at buses \#19, \#20 and \#21
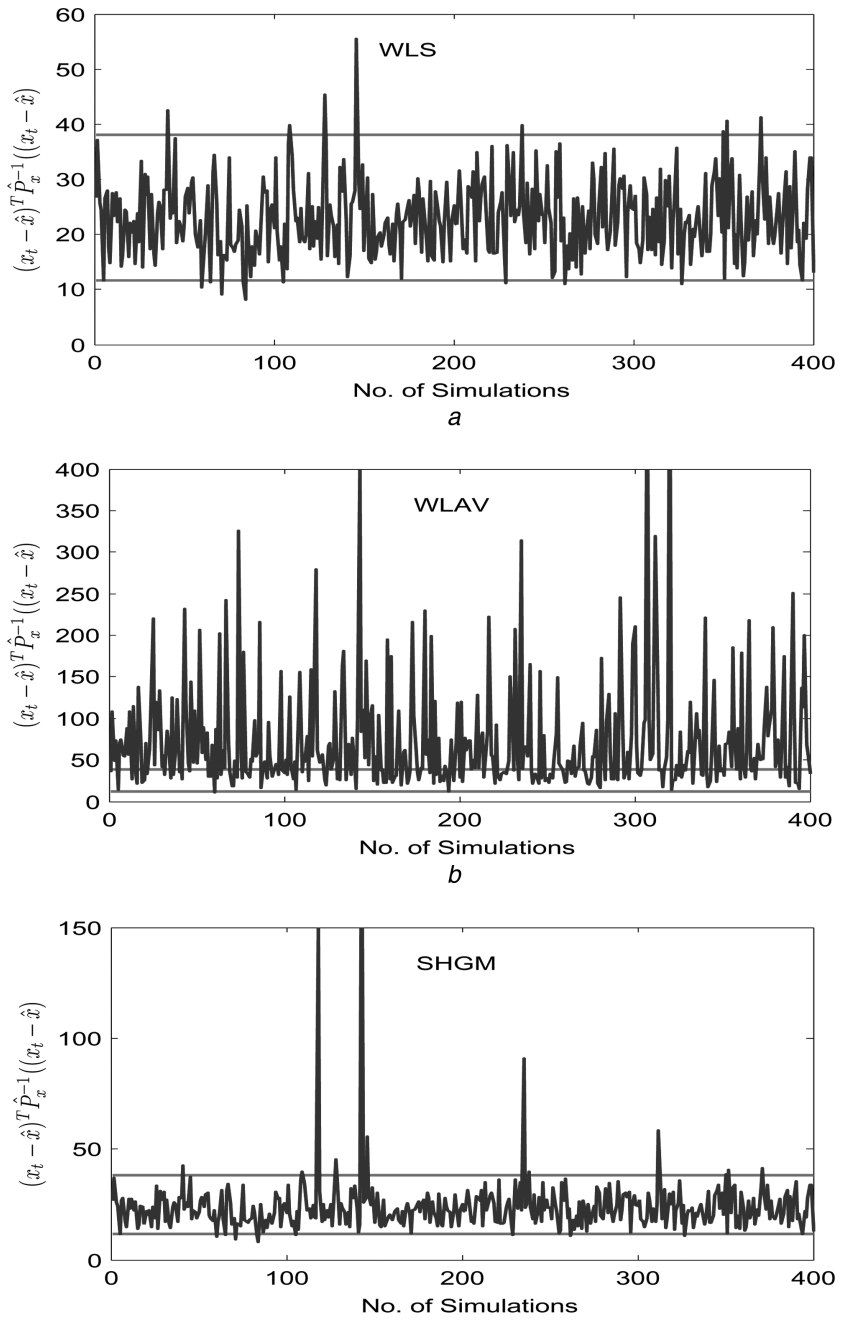

Figure 8 Twelve-bus system consistency plot: error in true measurements $=3 \%$, error in pseudo measurements $=50 \%$

The consistency plots for WLS and SHGM with increased redundancy are shown in Figs. 11 and 12, respectively. The WLS shows the consistent performance whereas the SHGM shows the inconsistency in all the simulated cases.

Table 3 Twelve-bus system performance summary

\begin{tabular}{|c|c|c|c|c|c|c|}
\hline \multirow[t]{2}{*}{ Estimator } & \multicolumn{3}{|c|}{ Real $1 \%$, pseudo $20 \%$} & \multicolumn{3}{|c|}{ Real $1 \%$, pseudo $50 \%$} \\
\hline & Bias & Consistent/E[ $\epsilon]$ & Quality $\operatorname{tr} /$ det & Bias & Consistent/E[ $\epsilon]$ & Quality tr/det \\
\hline WLS & unbiased & consistent/23.13 & $4.08 / 199.24$ & unbiased & consistent/23.25 & 3.7/179.01 \\
\hline WLAV & unbiased & inconsisten /136.7 & $3.17 / 191.46$ & unbiased & inconsistent/80.67 & $2.26 / 173.98$ \\
\hline \multirow[t]{2}{*}{ SHGM } & unbiased & inconsistent/1033.1 & $3.86 / 193.1$ & unbiased & consistent/26.37 & $3.7 / 178.28$ \\
\hline & \multicolumn{3}{|c|}{ Real 3\%, pseudo $20 \%$} & \multicolumn{3}{|c|}{ Real 3\%, pseudo 50\% } \\
\hline WLS & unbiased & consistent/23.85 & $1.89 / 198.4$ & unbiased & consistent/22.97 & $1.81 / 178.02$ \\
\hline WLAV & unbiased & inconsistent/130.91 & $1.68 / 190.53$ & unbiased & inconsistent/72.81 & $1.45 / 173.07$ \\
\hline SHGM & unbiased & inconsistent/953.8 & $1.86 / 192.37$ & unbiased & consistent/24.1 & $1.80 / 177.63$ \\
\hline
\end{tabular}



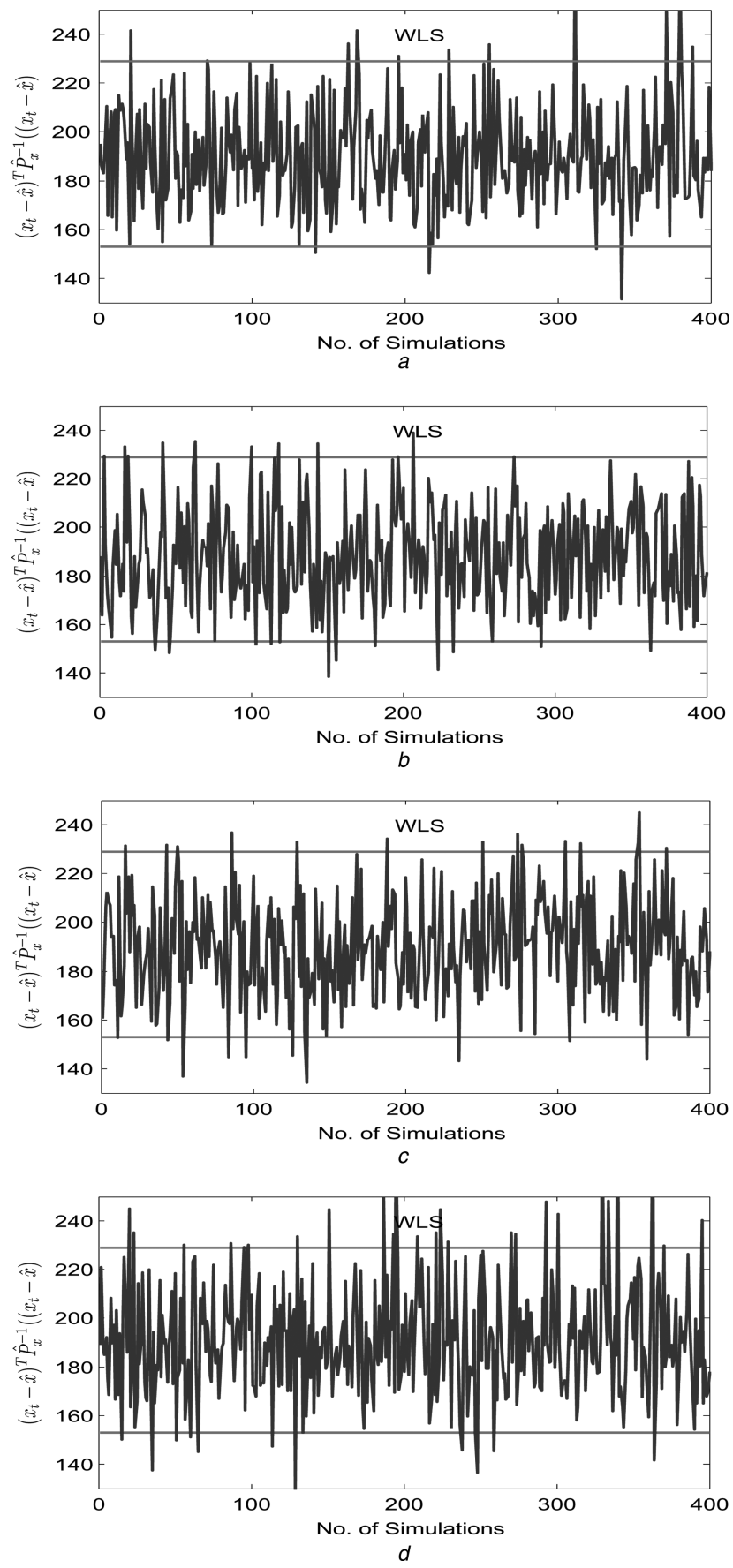

Figure 9 Ninety-five-bus system consistency plot with limited redundancy: WLS shows consistency in all test cases

A very high degree of inconsistency was observed in WLAV, which is difficult to show graphically.

The performance summaries for both cases are shown in Tables 4(a) and (b). In both the cases, the quality defined in (5) gives numerical instability in computations, hence it does not appear in the tables. Furthermore, the quality for WLAV estimator is inconsistent and shows negative values. This is because of very high variance of state estimates that are unacceptable for SE. In WLS and SHGM, as expected the qualities decrease with increase in errors in real and

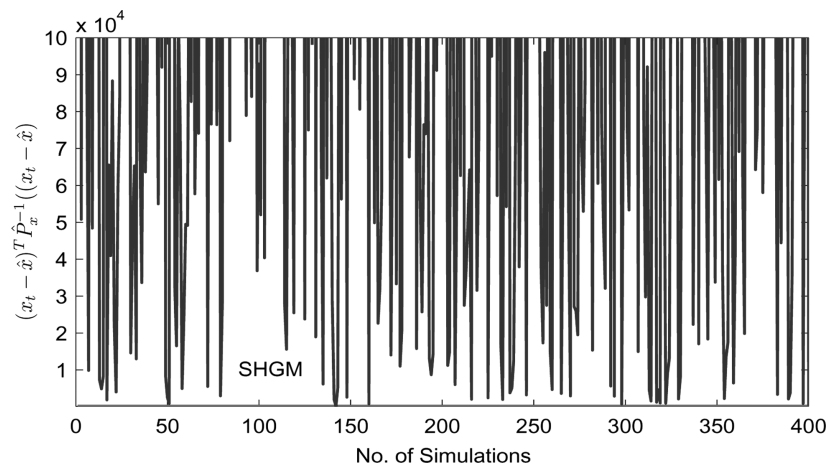

Figure 10 Ninety-five-bus system consistency plot with limited redundancy: error in true measurements $=1 \%$, error in pseudo measurements $=50 \%$

pseudo measurements. The value of $E[\epsilon]$ in the case of SHGM does not converge to the number of states (i.e. 189), which numerically verifies its inconsistency.

It is also important to note that with limited redundancy the trace qualities defined in (6) are close for both WLS and SHGM in Case 2 and Case 4. This gives the impression that SHGM should be consistent for these cases. Since trace captures the diagonal information of the error covariance matrix, it can be attributed that inconsistency in SHGM is mainly due to off-diagonal elements. In case of increased redundancy there is significant difference in the qualities of WLS and SHGM in all the test cases. The quality of WLS is better than the quality of SHGM.

In all the simulated cases only WLS satisfies the three statistical criteria (bias, consistency and quality) under the assumption of normal distribution of measurement errors. It can be concluded that the WLS is a suitable solver for the DSSE problem.

\subsection{Comments on error distribution and choice of solver}

The statistical criteria discussed in this paper depend on the characteristics of the distribution of measurement errors. The results presented are based on the assumption that the measurement errors are normally distributed. Under this assumption, the WLS satisfies the statistical criteria and hence was found to be the suitable solver for the SE. However, this may not be true if the measurement errors are not normally distributed. For instance if the errors follow the Laplace distribution [19], the WLAV estimator gives better performance than WLS and SHGM. The reason for this is that the WLAV is consistent with the Laplace distribution and maximisation of $\log$-likelihood of the Laplace density function results in the WLAV formulation. Hence, depending on the distribution of the errors, the corresponding statistical criterion discussed in Subsection 2.2 can be modified in order to identify the consistent solver for that distribution. 

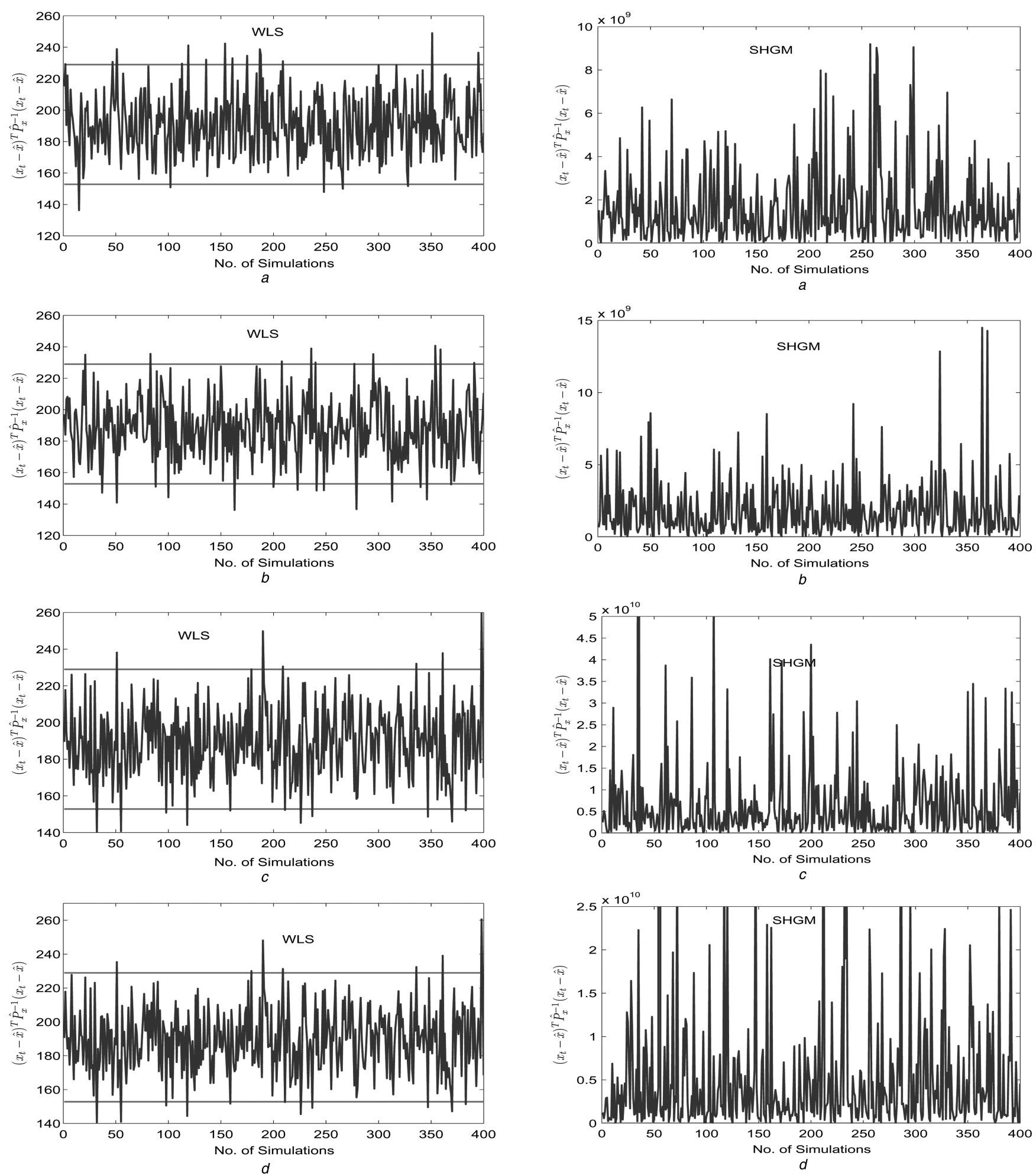

Figure 11 Ninety-five-bus system consistency plot with increased redundancy: WLS shows consistency in all the test cases

In reality, different probabilistic load distributions exist in the distribution networks and no standard distribution can fit all of them. Furthermore, the large size of the distribution network having various probability distributions at different buses makes accommodating them in a single state estimator

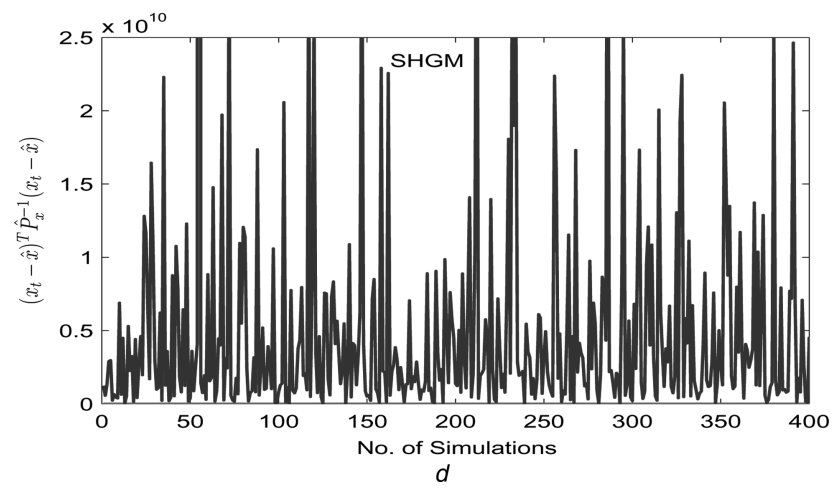

Figure 12 Ninety-five-bus system consistency plot with increased redundancy: SHGM shows inconsistency in all the test cases

impractical. A more practical approach is to model the actual probability distributions as a mixture of several Gaussian distributions (Fig. 13) and apply the WLS state estimator which is consistent with the normal distribution. This requires the modelling of the distribution of errors through 
Table 4 Ninety-five-bus UK-GDS performance summary

\begin{tabular}{|c|c|c|c|c|c|c|}
\hline \multirow{2}{*}{ Estimator } & \multicolumn{3}{|c|}{ Real 1\%, pseudo 20\% } & \multicolumn{4}{c|}{ Real 1\%, pseudo 50\% } \\
\cline { 2 - 7 } & Bias & Consistent $/ E[\epsilon]$ & Quality tr/det & Bias & Consistent $/ E[\epsilon]$ & Quality tr/det \\
\hline
\end{tabular}

(a) Limited redundancy

\begin{tabular}{|l|c|c|c|l|l|l|l|}
\hline WLS & unbiased & consistent $/ 190.02$ & $6.63 /-$ & unbiased & consistent $/ 188.16$ & $6.24 /-$ \\
\hline WLAV & unbiased & inconsistent $/ \infty$ & $-52 /-$ & unbiased & inconsistent $/ \infty$ & $-44.42 /-$ \\
\hline SHGM & unbiased & inconsistent $/ 3.06 \times 10^{4}$ & $6.46 /-$ & unbiased & inconsistent $/ 2.53 \times 10^{5}$ & $6.16 /-$ \\
\hline & \multicolumn{5}{|c|}{ Real 3\%, pseudo $20 \%$} & \multicolumn{4}{|c|}{ Real 3\%, pseudo 50\% } \\
\hline WLS & unbiased & consistent $/ 189.84$ & $4.61 /-$ & unbiased & consistent $/ 190.23$ & $4.41 /-$ \\
\hline WLAV & unbiased & Inconsistent $/ \infty$ & $-45.18 /-$ & unbiased & inconsistent $/ \infty$ & $-41.12 /-$ \\
\hline SHGM & unbiased & Inconsistent $/ 2.89 \times 10^{4}$ & $4.75 /-$ & unbiased & inConsistent $/ 2.27 \times 10^{5}$ & $4.4 /-$ \\
\hline
\end{tabular}

(b) Increased redundancy

\begin{tabular}{|l|c|c|c|l|l|c|}
\hline & \multicolumn{3}{|c|}{ Real 1\%, pseudo 20\% } & \multicolumn{3}{c|}{ Real 1\%, pseudo 50\% } \\
\hline WLS & unbiased & consistent $/ 190$ & $8.86 /-$ & unbiased & consistent $/ 188.3$ & $8.75 /-$ \\
\hline WLAV & unbiased & inconsistent $/ \infty$ & $-55.65 /-$ & unbiased & inconsistent $/ \infty$ & $-63.74 /-$ \\
\hline SHGM & unbiased & inconsistent $/ 1.65 \times 10^{9}$ & $6.70 /-$ & unbiased & inconsistent $/ 1.82 \times 10^{9}$ & $6.35 /-$ \\
\hline & \multicolumn{7}{|c|}{ Real 3\%, pseudo $20 \%$} & & \multicolumn{3}{c|}{ Real 3\%, pseudo 50\% } \\
\hline WLS & unbiased & consistent $/ 188.65$ & $6.85 /-$ & unbiased & consistent $/ 189.23$ & $6.75 /-$ \\
\hline WLAV & unbiased & inconsistent $/ \infty$ & $-43.73 /-$ & unbiased & inconsistent $/ \infty$ & $-49.88 /-$ \\
\hline SHGM & unbiased & inconsistent $/ 6.58 \times 10^{9}$ & $4.32 /-$ & unbiased & inconsistent $/ 1.0 \times 10^{10}$ & $4.28 /-$ \\
\hline
\end{tabular}

Gaussian mixture model (GMM) [20-22]. As shown in Fig. 13, the GMM represents an arbitrary distribution as a weighted combination of several Gaussian components. Mathematically, a GMM having $M_{\mathrm{c}}$ mixture components with mean and variance of $k$ th component as $\mu_{k}$ and $\sigma_{k}^{2}$ can be written as

$$
f(x)=\sum_{k=1}^{M_{c}} w_{k} \mathcal{N}\left(\mu_{k}, \sigma_{k}^{2}\right)(x) \text { and } \sum_{k=1}^{M_{c}} w_{k}=1
$$

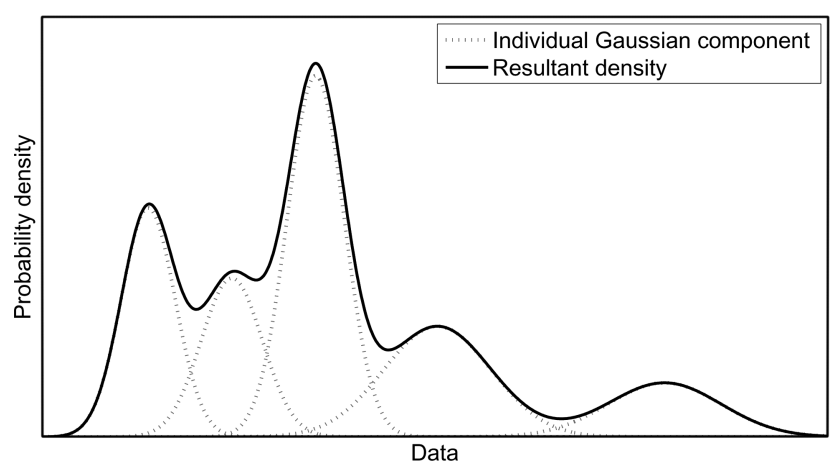

Figure 13 Gaussian mixture approximation of the density
The expectation maximisation algorithm [20-22] is used to obtain the parameters $\left(w_{k}, \mu_{k}, \sigma_{k}^{2}\right)$ of the GMM.

In transmission systems, all the estimators work well because of very high redundancy and thus the statistical measures to evaluate the performance are not required. For example, a highly erroneous measurement is treated as a bad data by the WLAV estimator and a redundant measurement is always available to replace this. But in distribution systems, the measurements are mainly the pseudo measurements with very limited redundancy. Since pseudo measurements are derived from the historical load profiles and customer behaviour, they are highly erroneous. This is why the statistical framework is required to identify the suitable solver for the DSSE.

\section{Conclusion}

The performance evaluation of SE techniques shows that the existing solution methodology of WLAV and SHGM cannot be applied to the distribution systems. In order to obtain the consistent and good quality estimate, significant modifications are required in these algorithms. WLS gives consistent and better quality performance when applied to distribution systems. Hence, WLS is found to be a suitable solver for the DSSE problem. 
The WLS works well if the noise characteristics are known. In the absence of this knowledge either the WLS needs to be modified or a new class of algorithms need to be introduced. Furthermore, with growing interest in the distribution automation, new DSSE techniques are expected to be introduced in the future. However, any modification in existing techniques or introduction of new algorithms should qualify some statistical criteria because of limited number of measurements. This paper highlights some important statistical criteria against which a SE algorithm should be tested to assess its suitability to DSSE.

\section{Acknowledgment}

The authors would like to thank Peter D. Lang of EDF Energy Networks for his valuable suggestions and discussions.

\section{References}

[1] ABUR A., EXPOSITO A.G.: 'Power system state estimation: theory and implementation' (Marcel Dekker, Inc., 2004)

[2] ShAFIU A., Jenkins t.V., StRBAC G.: 'Control of active networks', CIRED, 2005

[3] LU C.N., TENG J.H., LIU W.-H.E.: 'Distribution system state estimation', IEEE Trans. Power Syst., 1995, 10, (1), pp. $229-240$

[4] LIN W.-M., TENG J.-H.: 'Distribution fast decoupled state estimation by measurement pairing', IEE Proc.-Gener. Transm. Distrib., 1996, 143, (1), pp. 43-48

[5] BARAN M.E., KELLEY A.W.: 'A branch current based state estimation method for distribution systems', IEEE Trans. Power Syst., 1995, 10, (1), pp. 483-491

[6] WANG H., SCHULZ N.N.: 'A revised branch current based distribution system state estimation algorithm and meter placement impact', IEEE Trans. Power Syst., 2004, 19, (1), pp. 207-213

[7] LI K.: 'State estimation for power distribution system and measurement impacts', IEEE Trans. Power Syst., 1996, 11, (2), pp. 911-916

[8] BARAN M.E., ZHU J.X., Kelley A.W.: 'Meter placement for real time monitoring of distribution feeders', IEEE Trans. Power Syst., 1996, 11, (1), pp. 332-337

[9] GHOSH A.K., LUBKEMAN D.L., DOWNEY M.J., JONES R.H.: 'Distribution circuit state estimation using a probabilistic approach', IEEE Trans. Power Syst., 1997, 12, (1), pp. 45-51

[10] LUBKEMAN D.L., ZHANG J., GHOSH A.K., JONES R.H.: 'Field results for a distribution circuit state estimator implementation', IEEE Trans. Power Deliv., 2000, 15, (1), pp. 399-406
[11] BLACKMAN S.S.: 'Multiple target tracking with radar applications' (Canton St. Norwood: Artech House, Inc., 1986)

[12] ANDERSON T.W.: 'An introduction to multivariate statistical analysis' (Wiely, Seventh printing, 1966)

[13] JABR R.A.: 'Primal dual interior point approach to compute the $L_{1}$ solution of the state estimation problem', IEE Proc.Gener. Transm. Distrib., 2005, 152, (3), pp. 313-320

[14] HUBER P.J.: 'Robust statistics' (Wiely, 1981)

[15] CELIK M.K., LIU W.-H.E.: 'An incremental measurement placement algorithm for state estimation', IEEE Trans. Power Syst., 1995, 10, (3), pp. 1698-1703

[16] DAS D., NAGI H.S., KOTHARI D.P.: 'Novel method for solving radial distribution networks', IEE Proc.-Gener. Transm. and Distrib., 1994, 141, (4), pp. 291-298

[17] 'United Kingdom Generic Distribution Network (UKGDS)' [Online], available at: http://monaco.eee.strath.ac. uk/ukgds/, accessed June 2008

[18] SINGH R., PAL B.C., VINTER R.B.: 'Measurement placement in distribution system state estimation', IEEE Trans. Power Syst., 2009, 24, (2), pp. 668-675

[19] KOTZ S., KOZUBOWSKI T.J., PODGÓRSKI K.: 'The Laplace distribution and generalizations' (Birkhäuser Boston, 2001)

[20] DEMPSTER A.P., LAIRD N.M., RUBIN D.B.: 'Maximum-likelihood from incomplete data via the EM algorithm', J. R. Statist. Soc. Ser. B, 1977, 39, (1), pp. 1-38

[21] Redner R.A., WALKer H.F.: 'Mixture densities, maximum likelihood and the EM algorithm', SIAM Rev., 1984, 26, (2), pp. 195-239

[22] BILMES J.A.: 'A gentle tutorial on the EM algorithm and its application to parameter estimation for Gaussian mixture and hidden Markov models'. Technical Report, International Computer Science Institute, ICSI-TR-97-021, 1998

\section{Appendix}

\subsection{Computation of $\alpha$ for various estimators}

The fact that normalised measurement residual $r$ is normally distributed with zero mean and unit variance can be used to compute $\alpha$ for the state estimators discussed in Section 3.

\subsubsection{Weighted least-squares:}

$$
\psi(r)=r, \quad \psi^{\prime}(r)=1
$$




$$
\begin{gathered}
E\left[\psi^{2}(r)\right]=\frac{1}{\sqrt{2 \pi}} \int_{-\infty}^{\infty} r^{2} \mathrm{e}^{-(1 / 2) r^{2}} \mathrm{~d} r=\operatorname{Var}(r)=1 \\
E\left[\psi^{\prime}(r)\right]=\frac{1}{\sqrt{2 \pi}} \int_{-\infty}^{\infty} \mathrm{e}^{-(1 / 2) r^{2}} \mathrm{~d} r=1 \\
\alpha=\frac{E\left[\psi^{2}(r)\right]}{\left(E\left[\psi^{\prime}(r)\right]\right)^{2}}=1
\end{gathered}
$$

\subsubsection{Weighted least absolute value estimator:}

$$
\begin{gathered}
\psi(r)=\operatorname{sgn}(r), \psi^{\prime}(r)=2 \delta(r) \\
E\left[\psi^{2}(r)\right]=\frac{1}{\sqrt{2 \pi}} \int_{-\infty}^{\infty}(\operatorname{sgn}(r))^{2} \mathrm{e}^{-(1 / 2) r^{2}} \mathrm{~d} r=1
\end{gathered}
$$

We use the fact that

$$
\int_{-\infty}^{\infty} \delta\left(t-t_{0}\right) f(t) \mathrm{d} t=f\left(t_{0}\right)
$$

in the following expression

$$
\begin{gathered}
E\left[\psi^{\prime}(r)\right]=\frac{1}{\sqrt{2 \pi}} \int_{-\infty}^{\infty} 2 \delta(r) \mathrm{e}^{-(1 / 2) r^{2}} \mathrm{~d} r=\sqrt{\frac{2}{\pi}} \\
\alpha=\frac{E\left[\psi^{2}(r)\right]}{\left(E\left[\psi^{\prime}(r)\right]\right)^{2}}=\frac{\pi}{2}
\end{gathered}
$$

\subsubsection{Schweppe huber generalised M:}

$$
\begin{aligned}
& \psi(r)=\left\{\begin{array}{ccc}
r & \text { if } & |r| \leq a \omega \\
a \omega \operatorname{sgn}(r) & & \text { otherwise }
\end{array}\right. \\
& \psi^{\prime}(r)=\left\{\begin{array}{ccc}
1 & \text { if } & |r| \leq a \omega \\
2 a \omega \delta(r)=0 & & \text { otherwise }
\end{array}\right. \\
& E\left[\psi^{2}(r)\right]=\frac{1}{\sqrt{2 \pi}} \int_{-\infty}^{-a \omega}(a \omega \operatorname{sgn}(r))^{2} \mathrm{e}^{-(1 / 2) r^{2}} \mathrm{~d} r \\
& +\frac{1}{\sqrt{2 \pi}} \int_{-a \omega}^{a \omega} r^{2} \mathrm{e}^{-(1 / 2) r^{2}} \mathrm{~d} r \\
& +\frac{1}{\sqrt{2 \pi}} \int_{a \omega}^{\infty}(a \omega \operatorname{sgn}(r))^{2} \mathrm{e}^{-(1 / 2) r^{2}} \mathrm{~d} r
\end{aligned}
$$

By symmetry of the distribution the above equation can be expressed as

$$
=\frac{2}{\sqrt{2 \pi}} \int_{-\infty}^{-a \omega}(a \omega \operatorname{sgn}(r))^{2} \mathrm{e}^{-(1 / 2) r^{2}} \mathrm{~d} r+\frac{2}{\sqrt{2 \pi}} \int_{0}^{a \omega} r^{2} \mathrm{e}^{-(1 / 2) r^{2}} \mathrm{~d} r
$$

$$
=\left(2 a^{2} \omega^{2} \Phi(-a \omega)+\frac{2}{\sqrt{2 \pi}} \int_{0}^{a \omega} r^{2} \mathrm{e}^{-(1 / 2) r^{2}} \mathrm{~d} r\right)
$$

where $\Phi$ is the cumulative probability function. The integral term in the above equation is given by

$$
\frac{2}{\sqrt{2 \pi}} \int_{0}^{a \omega} r^{2} \mathrm{e}^{-(1 / 2) r^{2}} \mathrm{~d} r=-\sqrt{\frac{2}{\pi}} a \omega \mathrm{e}^{-\left(a^{2} \omega^{2} / 2\right)}+(2 \Phi(a \omega)-1)
$$

Using the relation $\Phi(-a \omega)=1-\Phi(a \omega)$ and substituting (35) in to (34), we obtain

$$
E\left[\psi^{2}(r)\right]=1-\sqrt{\frac{2}{\pi}} a \omega \mathrm{e}^{-\left(a^{2} \omega^{2} / 2\right)}+2\left(a^{2} \omega^{2}-1\right)(1-\Phi(a \omega))
$$

$$
E\left[\psi^{\prime}(r)\right]=\frac{1}{\sqrt{2 \pi}} \int_{-a \omega}^{a \omega} \mathrm{e}^{-(1 / 2) r^{2}} \mathrm{~d} r=2 \Phi(a \omega)-1
$$

In this case $\alpha$ depends on parameters ' $a$ ' and ' $\omega$ ', that is, if $a=1.5$ and $\omega=1$, the value of $\alpha$ is

$$
\alpha=\frac{E\left[\psi^{2}(r)\right]}{\left(E\left[\psi^{\prime}(r)\right]\right)^{2}}=\frac{0.7785}{(0.8664)^{2}}=1.0371
$$

